\title{
Application of Ga for Optimal Location and Parameters Setting Of Upfc Considering Voltage Stability Index
}

\author{
${ }^{1}$ K. Swathi ${ }^{2}$ K. Kiran Kumar \\ ${ }^{1} P G$ Scholar, Sree Vahini Institute of Science \& Technology, TIRUVURU, Krishna Dist, A.P., INDIA \\ ${ }^{2}$ HOD. of EEE, Sree Vahini Institute of Science \& Technology, TIRUVURU, Krishna Dist, A.P., INDIA
}

\begin{abstract}
In this paper the performance of UPFC is investigated in controlling the flow of power over the transmission line. Voltage sources model is utilized to study the behavior of the UPFC in regulating the voltage profile, active and reactive power. This model is incorporated in Conventional Newton Raphson algorithm for load flow studies. Simultaneous method is employed in which equations of unified power flow controller and the power balance equations of networks are combined in to one set of non-linear algebraic equations. Placement of this device in suitable location can lead to control in flow of power and maintain bus voltages in desired level and improve voltage stability margins. This Paper presents a Genetic Algorithm (GA) based allocation algorithm for UPFC Device considering energy cost, power system losses and cost of device. GA based method utilize the sensitivity of total real power transmission loss with respect to the control parameters of device. The results have been obtained on IEEE 5bus and IEEE 14bus test system.
\end{abstract}

Index Terms: Flexible AC Transmission Systems (FACTS), Power Flow Control, Unified Power Flow Controllers (UPFC), Newton-Raphson, Voltage Stability Index (VSI), Genetic Algorithm (GA).

\section{Introduction}

Flexible AC transmission system (FACTs) is an evolving technology based solution to help electric utilities fully utilize their transmission assets. Its first concept was introduced by N.G Hingorani, in 1988. Since then different kinds of FACTS devices have been proposed. Among them the Unified Power Flow controllers is the most versatile and effective device which was introduced in 1991[]. The UPFC consist of voltage source converters, one connected in series and other in shunt and both are connected back to back through a D.C capacitor .In order to investigate the impact of UPFC on power systems effectively, it is essential to formulate their correct and appropriate model. In the area of power flow analysis models of the UPFC have been published which treat the UPFC either as one series voltage source and one shunt current source model or both the series and the shunt are represented by voltage sources. Presented a decoupled model which is simple to implement but it presents some restrictions [2]. In [4] the UPFC is represented by two voltage sources called the voltage source model [3] discusses the distinguishing features of the voltage source model at length. [7] Introduced another model called the power injection models (PIM). Taking these two models as the base models, few other models have been developed with slight modifications in order to circumvent the limitations of the base models.

Due to ever increasing load demand and reduced rights of way, modern power transmission systems are forced to carry increasingly more power over long distances. Consequently, the transmission system becomes more stressed, which in turn, makes the system more vulnerable to voltage instability Voltage instability within the power system has serious consequences including voltage collapse and system blackout [1]. Voltage collapse is a process in which, the appearance of sequential events together with the voltage instability in a large area of system can lead to the case of unacceptable low voltage condition in the network, if no preventive measures are committed. Occurrence of a disturbance or load increasing can leads to excessive demand of reactive power. Therefore, system will show voltage instability. If additional resources provide sufficient reactive power support, the system will be established in a stable voltage level. However, sometimes there are not sufficient reactive power resources and the excessive demand of reactive power can leads to voltage collapse.

Application of FACTS devices is a very effective solution to prevent voltage instability and voltage collapse due to their fast and very flexible control. While FACTS devices is going to be located into transmission network, an important aspect is that the effectiveness of their damping is strongly influenced by their location [2].For a large-scale power system, more than one FACTS device may have to be installed in order to achieve the desired performance. Budgetary constraints force the utilities to limit the number of FACTS devices to be placed in a given system. Therefore, proper placement of these devices in the grid is an important issue. Voltage collapses are mostly initiated by a single disturbance (e.g. the outage of a line or a generator unit).So, to locate FACTS devices, consideration of contingency conditions is more important than consideration of normal state of the system. The drawbacks of several methods proposed in literature for the location of FACTS devices is that they only consider the normal state of the system [3, 4, and 5]. 
The proposed methodology (Genetic Algorithm) of optimal allocation of fixed UPFC for transmission network is implemented using MATLAB on the two test systems viz., IEEE 5-Bus and 14-Bus test systems. The cost constants and their typical values for this problem are energy cost $\left(K_{e}=3.5 \mathrm{Rs} / \mathrm{KWh}\right)$. The payback period assumed is ten years with depreciation factor $(\alpha)$ as 0.1 .

\section{Voltage Control of Power System}

A power system is said to be well designed if it gives a good quality of reliable supply. By good quality is meant the voltage levels within the reasonable limits. Practically all the equipment on the power systems are designed to operate satisfactorily only when the voltage levels on the system correspond to their rated voltages or at the most the variations are within say 5\%.If the voltage variation is more than a prescribed value, the performance of the equipments suffers and the life of most of the equipments suffers and the life of most of the equipment also is sacrificed. When power is supplied to a load through a transmission line keeping the sending end voltage constant, the receiving end or load voltage undergoes variations depending upon the magnitude of the load and the power factor of the load. The higher the load with smaller power factor the greater is the voltage variation. The voltage variation at a node is an indication of the unbalance between the reactive power generated and consumed by that node. If the reactive power generated is greater than consumed, the voltage goes up and vice versa. Whenever the voltage level of a particular bus undergoes variation this is due to the unbalance between the two versa at that bus

\section{Mathematical Model Of UPFC}

The Unified Power Flow Controller consists of two switching converter. These converters are operating from a common de link provided by a de storage capacitor as shown in Fig. 1.

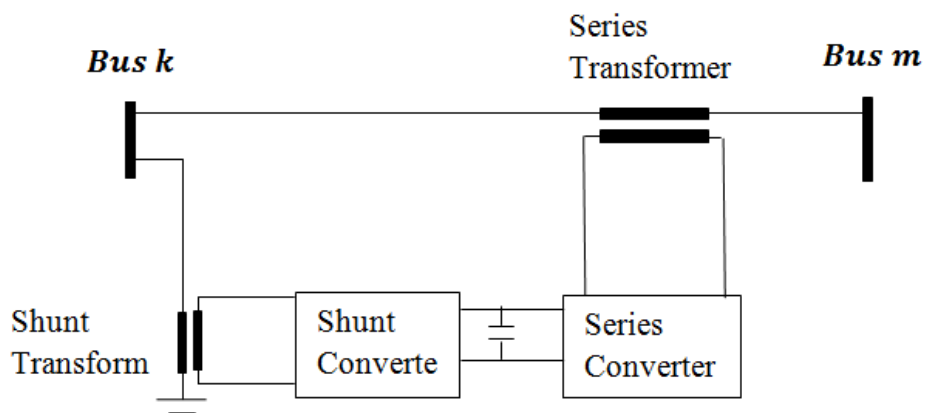

Fig. 1 Basic circuit arrangement of UPFC

Series Converter provides the main function of the UPFC by injecting an AC voltage with controllable magnitude and phase angle in series with the transmission line via a series transformer. The basic function of shunt converter is to supply or absorb the real power demand by series converter at the common dc link. It can also generate or absorb controllable reactive power and provide independent shunt reactive compensation for the line. Series converter supplies or absorbs locally the required reactive power and exchanges the active power as a result of the series injection voltage. The UPFC is new device in FACTS family which consists of series and shunt connected converter. The UPFC can provide the necessary functional flexibility for power flows control. This approach allows the combined application of phase angle with controlled series and shunt reactive compensation. The UPFC has the regulating the power flow and minimizing the losses at the same time.

A schematic representation of a UPFC is shown in Fig. 2. The output voltage of the series converter is added to the AC terminal voltage $V_{0}$ via the series connected coupling transformer. The injected voltage $V_{c R}$ acts as an AC series voltage source, changing the effective sending-end voltage as seen from node $m$. The product of the transmission line current $I_{m}$ and the series voltage source $V_{c R}$, determines the active and reactive power exchanged between the series converter and the AC system. The real power demanded by the series converter is supplied from the AC power system by the shunt converter via the common DC link. The shunt converter is able to generate or absorb controllable reactive power in both operating modes (i.e., rectifier and inverter). The independently controlled shunt reactive compensation can be used to maintain the shunt converter terminal AC voltage magnitude at a specified value.

The UPFC equivalent circuit shown in Fig. 2 is used to device the steady-state model. 


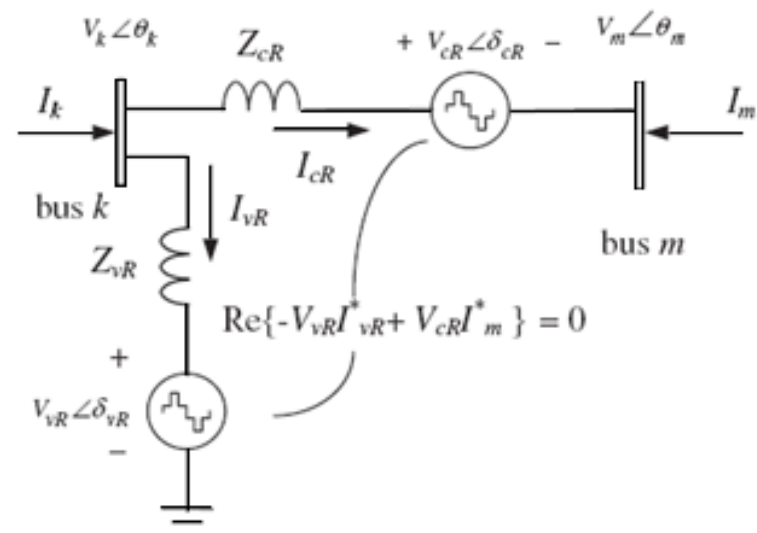

Fig. 2 Unified Power Flow Controller equivalent circuit

The equivalent circuit consists of two ideal voltage sources representing the fundamental Fourier series component of the switched voltage waveforms at the AC converter terminals. The ideal voltage sources are:

$$
\begin{aligned}
& V_{v R}=V_{v R}\left(\cos \theta_{v R}+\sin \theta_{v R}\right) \\
& V_{c R}=V_{c R}\left(\cos \theta_{c R}+\sin \theta_{c R}\right)
\end{aligned}
$$

Where $V_{v R}$ and $\theta_{v R}$ are the controllable magnitude $\left(V_{v R \min } \leq V_{v R} \leq V_{v R \max }\right)$ and angle $\left(0 \leq \theta_{v R} \leq 2 \pi\right)$ of the voltage source representing the shunt converter. The magnitude $V_{c R}$ and angle $\theta_{c R}$ of the voltage sources of the series converter are controlled between limits $\left(V_{c R \min } \leq V_{c R} \leq V_{c R \max }\right)$ and $\left(0 \leq \theta_{c R} \leq 2 \pi\right)$, respectively.

\section{UPFC Power And Jacobian Equations:} At node $k$ :

Based on the equivalent circuit shown in Fig. 2. the active and reactive power equations are

$$
\begin{aligned}
P_{k}=V_{k}^{2} G_{k k}+ & V_{k} \\
& V_{m}\left(G_{k m} \cos \left(\theta_{k}-\theta_{m}\right)\right. \\
& \left.+B_{k m} \sin \left(\theta_{k}-\theta_{m}\right)\right) \\
& +V_{k} V_{c R}\left(G_{k m} \cos \left(\theta_{k}-\theta_{c R}\right)\right. \\
& \left.+B_{k m} \sin \left(\theta_{k}-\theta_{c R}\right)\right) \\
& +V_{k} V_{v R}\left(G_{v R} \cos \left(\theta_{k}-\theta_{v R}\right)+B_{v R} \sin \left(\theta_{k}-\theta_{v R}\right)\right) \\
Q_{k}=-V_{k}^{2} B_{k k}+ & V_{k} V_{m}\left(G_{k m} \sin \left(\theta_{k}-\theta_{m}\right)\right. \\
& \left.-B_{k m} \cos \left(\theta_{k}-\theta_{m}\right)\right) \\
& +V_{k} V_{c R}\left(G_{k m} \sin \left(\theta_{k}-\theta_{c R}\right)\right. \\
& \left.-B_{k m} \cos \left(\theta_{k}-\theta_{c R}\right)\right)+V_{k} V_{v R}\left(G_{v R} \sin \left(\theta_{k}-\theta_{v R}\right)-B_{v R} \cos \left(\theta_{k}-\theta_{v R}\right)\right)
\end{aligned}
$$

At node $m:$

$$
\begin{aligned}
P_{m}=V_{m}^{2} G_{m m}+ & V_{k} V_{m}\left(G_{m k} \cos \left(\theta_{m}-\theta_{k}\right)\right. \\
& \left.+B_{m k} \sin \left(\theta_{m}-\theta_{k}\right)\right)+V_{m} V_{c R}\left(G_{m m} \cos \left(\theta_{m}-\theta_{c R}\right)+B_{m m} \sin \left(\theta_{m}-\theta_{c R}\right)\right) \\
Q_{m}=-V_{m}^{2} B_{m m} & +V_{k} V_{m}\left(G_{m k} \sin \left(\theta_{m}-\theta_{k}\right)\right. \\
& \left.-B_{m k} \cos \left(\theta_{m}-\theta_{k}\right)\right)+V_{m} V_{c R}\left(G_{m m} \sin \left(\theta_{m}-\theta_{c R}\right)-B_{m m} \cos \left(\theta_{m}-\theta_{c R}\right)\right)
\end{aligned}
$$

Series converter:

$$
\begin{aligned}
& P_{c R}=V_{c R}^{2} G_{m m}+V_{c R} V_{k}\left(G_{k m} \cos \left(\theta_{c R}-\theta_{k}\right)+B_{k m} \sin \left(\theta_{c R}-\theta_{k}\right)\right)+ \\
& V_{c R} V_{m}\left(G_{m m} \cos \left(\theta_{c R}-\theta_{m}\right)+B_{m m} \sin \left(\theta_{c R}-\theta_{v R}\right)\right) \\
& Q_{c R}=-V_{c R}^{2} B_{m m}+V_{k} V_{c R}\left(G_{k m} \sin \left(\theta_{c R}-\theta_{k}\right)\right. \\
& \left.-B_{k m} \cos \left(\theta_{c R}-\theta_{k}\right)\right) \\
& +V_{m} V_{c R}\left(G_{m m} \sin \left(\theta_{c R}-\theta_{m}\right)\right. \\
& \left.-B_{m m} \cos \left(\theta_{c R}-\theta_{m}\right)\right) \\
& \text { Shunt converter: } P_{v R}=-V_{v R}^{2} G_{v R}+V_{v R} V_{k}\left(G_{v R} \cos \left(\theta_{v R}-\theta_{k}\right)+B_{v R} \sin \left(\theta_{v R}-\theta_{k}\right)\right) \\
& Q_{v R}=V_{v R}^{2} B_{v R}+V_{v R} V_{k}\left(G_{v R} \sin \left(\theta_{v R}-\theta_{k}\right)-B_{v R} \cos \left(\theta_{v R}-\theta_{k}\right)\right)
\end{aligned}
$$

The general transfer admittance matrix for the UPFC is obtained by applying Kirchhoff current and voltage laws to the electric circuit shown in Fig. 2. and given by

$$
\left[\begin{array}{c}
I_{k} \\
I_{m}
\end{array}\right]=\left[\begin{array}{cccc}
Y_{k k} & Y_{k m} & Y_{k m} & Y_{v R} \\
Y_{m k} & Y_{m m} & Y_{m m} & 0
\end{array}\right]\left[\begin{array}{c}
V_{k} \\
V_{m} \\
V_{c R} \\
V_{v R}
\end{array}\right]
$$

Where $y_{c R}=\frac{1}{z_{c R}}=\frac{1}{R_{c R}+j X_{c R}}$ 


$$
\begin{gathered}
y_{v R}=\frac{1}{z_{v R}}=\frac{1}{R_{v R}+j X_{v R}} \\
Y_{k k}=G_{k k}+j B_{k k}=y_{c R}+y_{v R} \\
Y_{m m}=G_{m m}+j B_{m m}=y_{c R} \\
Y_{k m}=Y_{m k}=G_{k m}+j B_{k m}=-y_{c R} \\
Y_{v R}=G_{v R}+j B_{v R}=-y_{v R}
\end{gathered}
$$

Assuming a loss-free converter operation, the UPFC neither absorbs nor injects active power with respect to the AC system. The active power demanded by the series converter is supplied from the AC power system by the shunt converter via the common DC link. The Dc link voltage, $V_{d c}$, remains constant. Hence, the active power supplied to the shunt converter $P_{v R}$, must satisfy the active power demanded by the series converter, $P_{C R}$, i.e.,

$$
\begin{gathered}
P_{c R}+P_{v R}=0 \\
\text { Where } P_{c R}=V_{c R}^{2} G_{m m}+V_{c R} V_{k}\left(G_{k m} \cos \left(\theta_{c R}-\theta_{k}\right)+B_{k m} \sin \left(\theta_{c R}-\theta_{k}\right)\right)+ \\
V_{c R} V_{m}\left(G_{m m} \cos \left(\theta_{c R}-\theta_{m}\right)+B_{m m} \sin \left(\theta_{c R}-\theta_{v R}\right)\right) \\
P_{v R}=-V_{v R}^{2} G_{v R}+V_{v R} V_{k}\left(G_{v R} \cos \left(\theta_{v R}-\theta_{k}\right)+B_{v R} \sin \left(\theta_{v R}-\theta_{k}\right)\right)
\end{gathered}
$$

Also, by assuming a loss-free coupling transformer operation, the active power at node $k, P_{k}$, should match the active power at node $m, P_{m}$. Then, an alternative equation which satisfies the constant $V_{d c}$, constant is

$$
P_{k}+P_{m}=0
$$

The UPFC linearized power equation are combined with the linearized system of equation corresponding to the rest of the network,

$$
[g(X)]=[J][\Delta X]
$$

Where $[g(X)]=\left[\begin{array}{llllll}\Delta P_{k} & \Delta P_{m} & \Delta Q_{k} & \Delta Q_{m} \Delta P_{m k} & \Delta Q_{m k} & P_{c R}+P_{v R}\end{array}\right]^{T}$

The superscript $\mathrm{T}$ indicates transposition. $[\Delta X]$ is the solution vector and $[J]$ is the Jacobian matrix. If both nodes, $k$ and $m$, are PQ- type and the UPFC is controlling active power, flowing from $m$ to $k$, and reactive power injected at node $m$, the solution vector and the Jacobin matrix are defined as shown in Eqns. (16) and (17) . Assuming the power control mentioned above and that the UPFC controls voltage magnitude at the AC system shunt converter terminal (node $k$ ), the solution vector and the Jacobian matrix are shown in Eqns. (18) and (19).

$$
\begin{aligned}
& \begin{array}{c}
{[\Delta X]=\left[\begin{array}{lllllll}
\Delta \theta_{k} & \Delta \theta_{m} & \frac{\Delta V_{k}}{V_{k}} & \frac{\Delta V_{m}}{V_{m}} & \Delta \theta_{c R} & \frac{\Delta V_{c R}}{V_{c R}} & \Delta \theta_{v R}
\end{array}\right]^{T}} \\
{[J]=\left[\begin{array}{ccccccc}
H_{k k} & H_{k m} & N_{k k} & N_{k m} & H_{k c R} & N_{k c R} & H_{c v R} \\
H_{m k} & H_{m m} & N_{m k} & N_{m m} & H_{m v R} & N_{m v R} & 0 \\
J_{k k} & J_{k m} & L_{k k} & L_{k m} & J_{k c R} & L_{k c R} & J_{k v R} \\
J_{m k} & J_{m m} & L_{m k} & L_{m m} & J_{m c R} & L_{m c R} & 0 \\
H_{m k} & H_{m m} & N_{m k} & N_{m m} & H_{m c R} & N_{m c R} & 0 \\
J_{m k} & J_{m m} & L_{m k} & L_{m m} & J_{m c R} & L_{m c R} & 0 \\
H_{c R k}+H_{v R k} & H_{c R m} & H_{c R k}+N_{v R k} & N_{c R m} & H_{c R c R} & N_{c R c R} & H_{v R v R}
\end{array}\right] .}
\end{array} \\
& {[\Delta X]=\left[\begin{array}{lllllll}
\Delta \theta_{k} & \Delta \theta_{m} & \frac{\Delta V_{v R}}{V_{v R}} & \frac{\Delta V_{m}}{V_{m}} & \Delta \theta_{c R} & \frac{\Delta V_{c R}}{V_{c R}} & \Delta \theta_{v R}
\end{array}\right]^{T}} \\
& {[J]=\left[\begin{array}{ccccccc}
H_{k k} & H_{k m} & N_{k v R} & N_{k m} & H_{k c R} & N_{k c R} & H_{c v R} \\
H_{m k} & H_{m m} & 0 & N_{m m} & H_{m v R} & N_{m v R} & 0 \\
J_{k k} & J_{k m} & L_{k v R} & L_{k m} & J_{k c R} & L_{k c R} & J_{k v R} \\
J_{m k} & J_{m m} & 0 & L_{m m} & J_{m c R} & L_{m c R} & 0 \\
H_{m k} & H_{m m} & 0 & N_{m m} & H_{m c R} & N_{m c R} & 0 \\
J_{m k} & J_{m m} & 0 & L_{m m} & J_{m c R} & L_{m c R} & 0 \\
H_{c R k}+H_{v R k} & H_{c R m} & N_{c R k}+N_{v R k} & N_{c R m} & H_{c R c R} & N_{c R c R} & H_{v R v R}
\end{array}\right]}
\end{aligned}
$$

\section{Voltage Stability Index}

The voltage stability index or proximity is the device used to indicate the voltage stability condition formulated based on a line or a bus [8]. The proposed method builds on recent advanced in the areas of real-time voltage stability monitoring and control. The maximum threshold is set at unity as the maximum value beyond which this limit system bifurcation will be experienced.

Consider an $n$-bus system having $1,2,3, \ldots n$, generator buses $(g)$, and $g+1, g+2, \ldots n$, the load buses $(r=$ $n-g-s)$. The transmission system can be represented by using a hybrid representation, by the following set of equations 


$$
\left[\begin{array}{l}
V_{L} \\
I_{G}
\end{array}\right]=H\left[\begin{array}{c}
I_{L} \\
V_{L}
\end{array}\right]=\left[\begin{array}{ll}
Z_{L L} & F_{L G} \\
K_{G L} & Y_{G G}
\end{array}\right]\left[\begin{array}{c}
I_{L} \\
V_{G}
\end{array}\right]
$$

It can be seen that when a load bus approaches a steady state voltage collapse situation, the index $L$ approaches the numerical value 1.0. Hence for an overall system stability condition, the index evaluated at any of the buses must be less than unity. Thus the index value $L$ gives an indication of how far the system is from voltage collapse. The $L-$ indices for a given load condition are computed for all load buses. The equation for the $L$-index for $j^{\text {th }}$ node can be written as,

$$
\begin{array}{r}
L_{j}=\left|1-\sum_{i=1}^{i=g}\right| F_{j i}\left|\frac{\left|V_{i}\right|}{\left|V_{j}\right|}\left(F_{j i}^{r}+j F_{j i}^{m}\right)\right| \\
\mathrm{F}_{\mathrm{ji}}^{\mathrm{m}}=\left|\mathrm{F}_{\mathrm{ji}}\right| \sin \left(\theta_{\mathrm{ji}}^{\mathrm{r}}=\left|\delta_{\mathrm{ji}}\right| \cos \left(\delta_{\mathrm{i}}-\delta_{\mathrm{ji}}\right)\right.
\end{array}
$$

It can be seen that when a load bus approaches a steady state voltage collapse situation, the index $L$ approaches the numerical value 1.0. Hence for an overall system voltage stability condition, the index evaluated at any of the buses must be less than unity. Thus the index value $L$ gives an indication of how far the system is from voltage collapse.

\section{Evolutionary Optimization Technique (Ga)}

Genetic Algorithm (GA) is one of the most famous meta-heuristic optimization algorithms which is based on natural evolution and population. Genetics which is usually used to reach to near global optimum solution. In each iteration of GA (referred as generation), a new set of string (i.e. chromosomes) with improved fitness is produced using genetic operators (i.e. selection, crossover and mutation) [13], [14].

Selection Operator: Key idea: give preference to better individuals, allowing them to pass on their genes to the next generation. The goodness of each individual depends on its fitness. Fitness may be determined by an objective function or by a subjective judgment

Crossover Operator: Prime distinguished factor of GA from other optimization techniques. Two individuals are chosen from the population using the selection operator .A crossover site along the bit strings is randomly chosen. The values of the two strings are exchanged up to this point. If $S 1=000000$ and $s 2=111111$ and the crossover point is 2 then $\mathrm{S}^{\prime}=110000$ and $\mathrm{s}^{\prime}=001111$. The two new offspring created from this mating are put into the next generation of the population.By recombining portions of good individuals, this process is likely to create even better individuals

Mutation Operator: With some low probability, a portion of the new individuals will have some of their bits flipped. Its purpose is to maintain diversity within the population and inhibit premature convergence. Mutation alone induces a random walk through the search space; Mutation and selection (without crossover) create a parallel, noise-tolerant, hill-climbing algorithm..

The algorithm consists of the following steps:

Begain

initialize

chromosomes in the population

evaluate fitness of all chromosomes

do until

number of generation is large enough

do until

the new population if formed

select parents from the old population

produce offspring's via reproduction, crossover or mutation process

evaluate fitness of offspring's

end do

end do

end .

Using Siemens AG Database, cost function for UPFC is developed as follows:

$$
C_{U P F C}=0.0003 S^{2}-0.2691 S+188.22 U S \$ / k V A R
$$

Where, $S$ is operating range of UPFC in MVAR

$S=\left|Q_{2}-Q_{1}\right|$

$Q_{1}$ - MVAR flow through the branch before placing FACTS device. 
Application of Ga for Optimal Location and Parameters Setting Of Upfc Considering Voltage..

$Q_{2} \quad$ - MVAR flow through the branch after placing FACTS device.

The goal of optimization algorithm is to place FACTS devices in order to enhance voltage stability margin of power system considering cost function FACTS devices. So these devices should be place to prevent congestion in transmission lines and transformer and maintain bus voltages close to their reference.

Fitness function is expressed as below:

Max. $f=\left\{K e \times T \times[T L-U P F C T L]-\alpha \times\left[C_{\text {upfc }} \times U P F C_{\text {rating }}\right]\right\}$.

\section{Simulation Results}

The proposed methodology of optimal allocation of fixed UPFC for transmission network is implemented using MATLAB on the two test systems viz., IEEE 5-Bus and 14-Bus test systems. The cost constants and their typical values for this problem are energy cost $\left(K_{e}=3.5 \mathrm{Rs} / \mathrm{KWh}\right)$. The payback period assumed is ten years with depreciation factor $(\alpha)$ as 0.1 .

\section{IEEE 5-Bus test system}

A small network is used illustrate the power flows solution given by Newton-Raphson method. As shown in Fig. 3, five-bus network containing two generator and seven transmission lines.

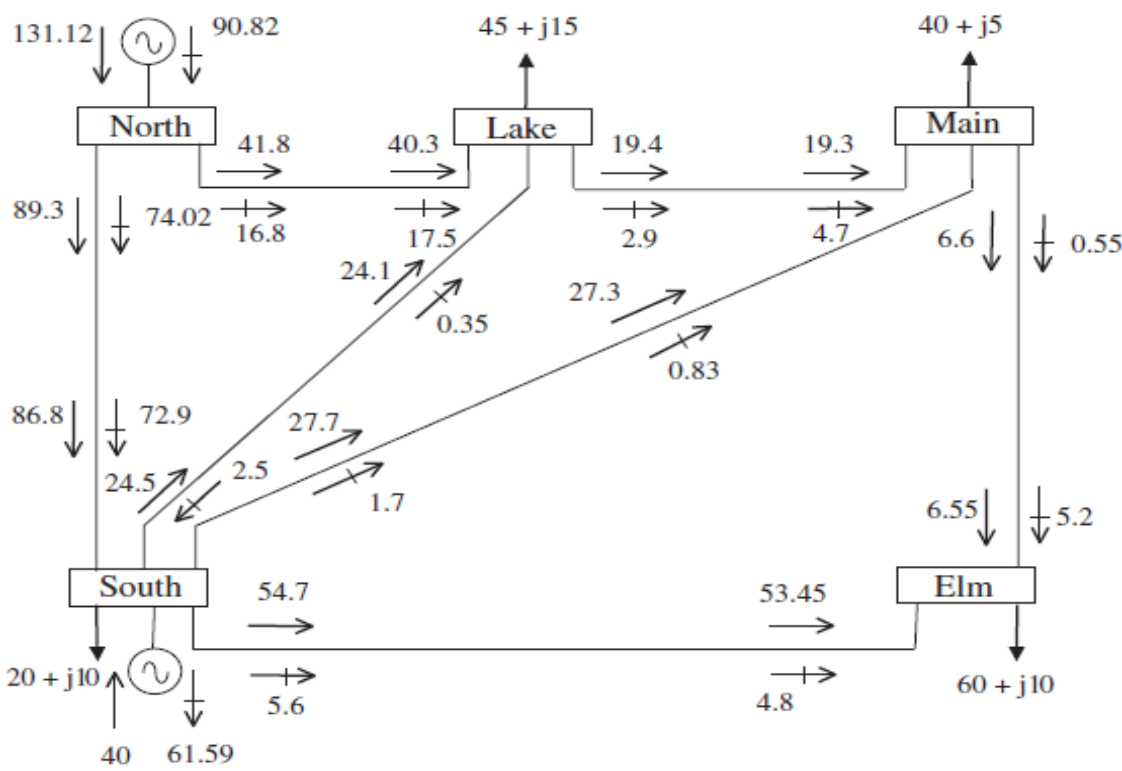

Fig. 3. The IEEE 5-Bus test network and power flow results

The largest power flow takes place in the transmission line-1 connecting the two generator busses: 89.33 MW \& 74.00 MVAR leave the North Bus and 86.85 MW \& 72.91 MVAR arrives at the South Bus as shown in Fig. 3. This is also the transmission line (i.e., line-1) that incurs higher active power loss $2.48 \mathrm{MW}$. The total power loss is 12.395 MVA. The operating conditions demand a large amount of reactive power generation by the generator connected at North Bus (i.e., 90.82 MVAR). This amount is well in excess of the reactive power drawn by the system loads (i.e., 40MVAR). The generator at South Bus draws the excess of reactive power in network (i.e., $61.59 \mathrm{MVAR}$ ). This amount includes the net reactive power produced by the several of the transmission lines. For the above discussed network, the existed method and proposed method are applied, presented as case (i) and case (ii). The numerical results and discussion are as follows.

\section{Case (i): Existed Method (UPFC Located at Lake Bus)}

The five-bus network is modified to include one UPFC to compensate the transmission line link bus Lake and bus Main as given. The UPFC is used to maintain active and reactive powers leaving the UPFC, towards Main at $40 \mathrm{MW}$ and 2 MVAR, respectively. Moreover the UPFC shunt converter is set to regulate the nodal voltage magnitude at Lake at $1 \mathrm{p}$. u. The starting values of the UPFC voltage sources are taken to be $V_{c R}=0.04 p . u, \delta_{c R}=87.13^{0}, V_{v R}=1 p . u$ and $\delta_{v R}=0^{0}$. Information on how to determine the starting values for these voltage sources is given in. The source impedances have values of $X_{c R}=X_{v R}=0.1 p$. u. Convergence is obtained in five iteration to a power mismatch tolerance $1 e^{-12}$. The UPFC held its target values. The power flow results are shown in Fig. 4. 
Application of Ga for Optimal Location and Parameters Setting Of Upfc Considering Voltage..

As expected, the power flows in the UPFC upgraded network differ with respect to the original case. The most noticeable changes are as follows: there is a $32 \%$ increase of active power flowing towards Lake through transmission lines North-Lake and South-Lake. The increase is in response to the large amount of active power demanded by the UPFC series converter. The maximum amount of active power exchange between the UPFC and the AC system will depend on the robustness of the UPFC shunt bus, Lake. Since the UPFC generates its own reactive power, the generators at decrease its reactive power generation by $5.6 \%$, and the generator connected at South increase its absorption of reactive power by $22.6 \%$.

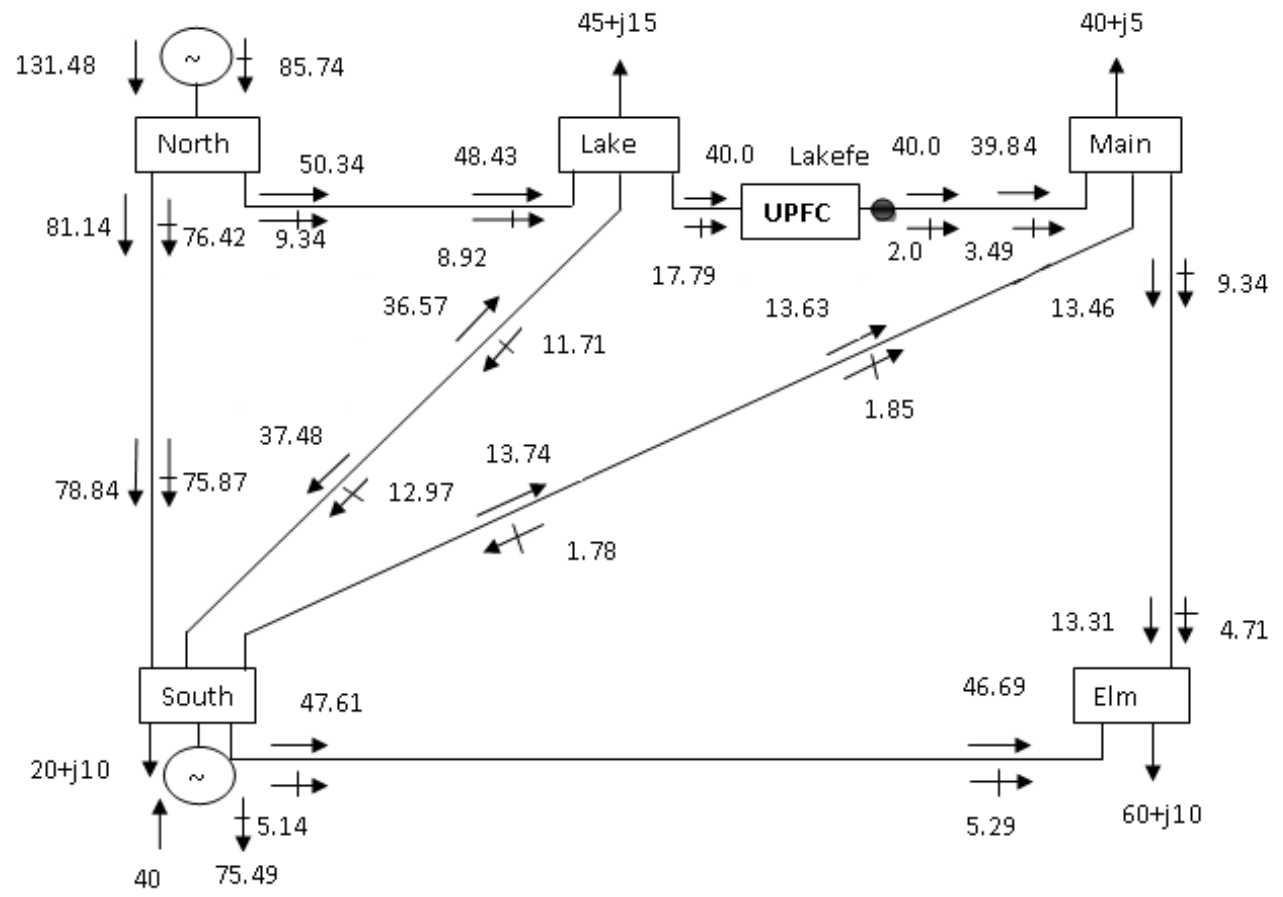

Fig. 4 The five-bus test network with one UPFC at Lake Bus, and the power flow results

Case (ii): Proposed Method (UPFC Located at Bus Elm)

Voltage stability indices are calculated for the IEEE 5-Bus system without any FACTS devices

Table 1 Voltage Stability Index before UPFC device

\begin{tabular}{|l|l|}
\hline Load Bus & Volateg stability indices $(\mathrm{Lj})$ in p.u \\
\hline Lake & 0.3535 \\
\hline Main & 0.3584 \\
\hline Elm & $\mathbf{0 . 3 9 1 3}$ \\
\hline
\end{tabular}

By considering the above Table 1, it is observed that bus Elm is more sensitive towards system security. Therefore bus Elm is more suitable location for UPFC to improve power system security/stability. An additional node is termed as node Elmfa, is used to connect the UPFC. The modified original network is including a UPFC between nodes Elm and Elmfa as shown in Fig. 4. After placing UPFC voltage stability index of the system is presented in Table 2.

Table 2 Voltage Stability Index After UPFC device

\begin{tabular}{|l|l|}
\hline Load Bus & Voltage stability indices $(\mathrm{Lj})$ in p.u \\
\hline Lake & 0.2936 \\
\hline Main & 0.2831 \\
\hline Elm & 0.0190 \\
\hline Elmfa & 0.1181 \\
\hline
\end{tabular}

The UPFC is used to maintain active and reactive powers leaving the UPFC, towards Main at 65.6 MW and 5.17 MVARs, respectively. Moreover, the UPFC's shunt converter is set to regulate Elm's nodal voltage magnitude at 1 p. u. The initial conditions of the UPFC voltage sources are computed by using equation given in Section 2.3, $V_{c R}=0.008352$ p. u, $\theta_{c R}=-51.758^{0}, V_{v R}=1$ p.u and $\theta_{v R}=0^{0}$. 


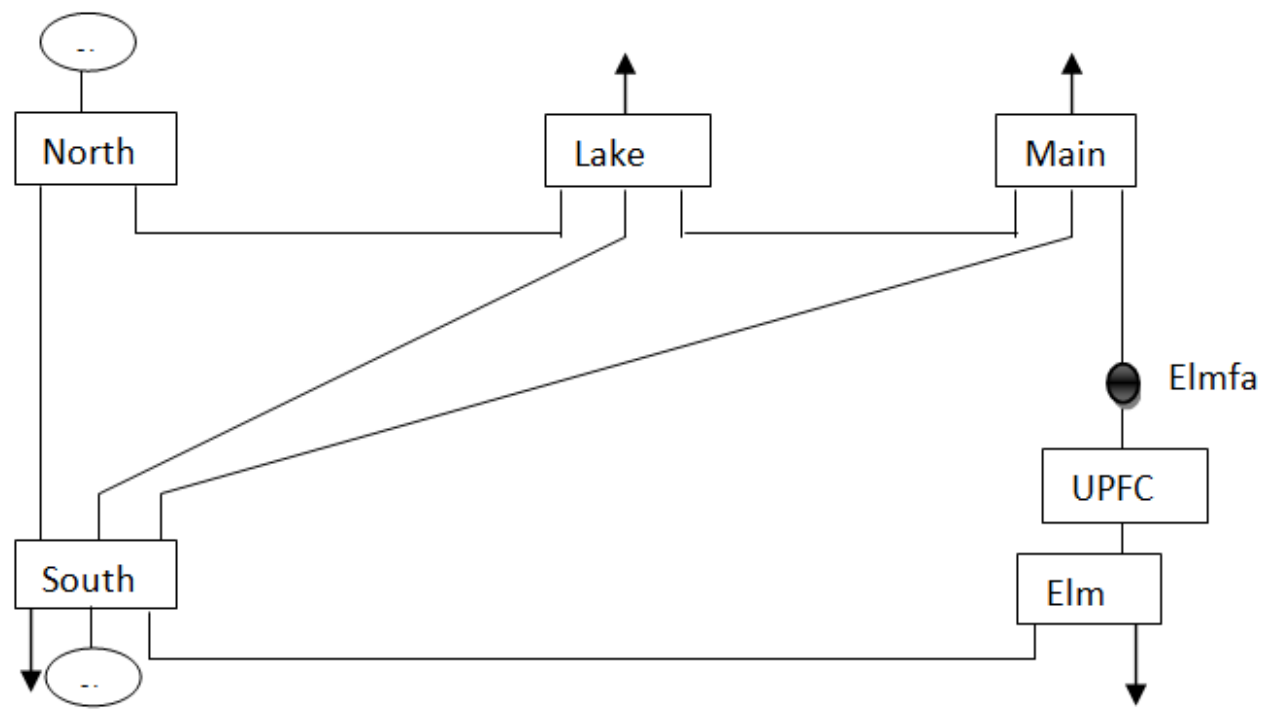

Fig. 4 Modified original network

The source impedances have values of $X_{c R}=X_{v R}=0.1$ p.u. The UPFC upheld its target values. The power flow results are shown in Fig. 5. As expected, there are 25 percentage increases of active power flowing towards Elm through transmission lines via South-Elm. The increase is in response to the large amount of active power demanded by UPFC series converter. Since the UPFC generates its own reactive power, the generator North decreases its reactive power generation by $4.9 \%$ and the voltage profile is increased.

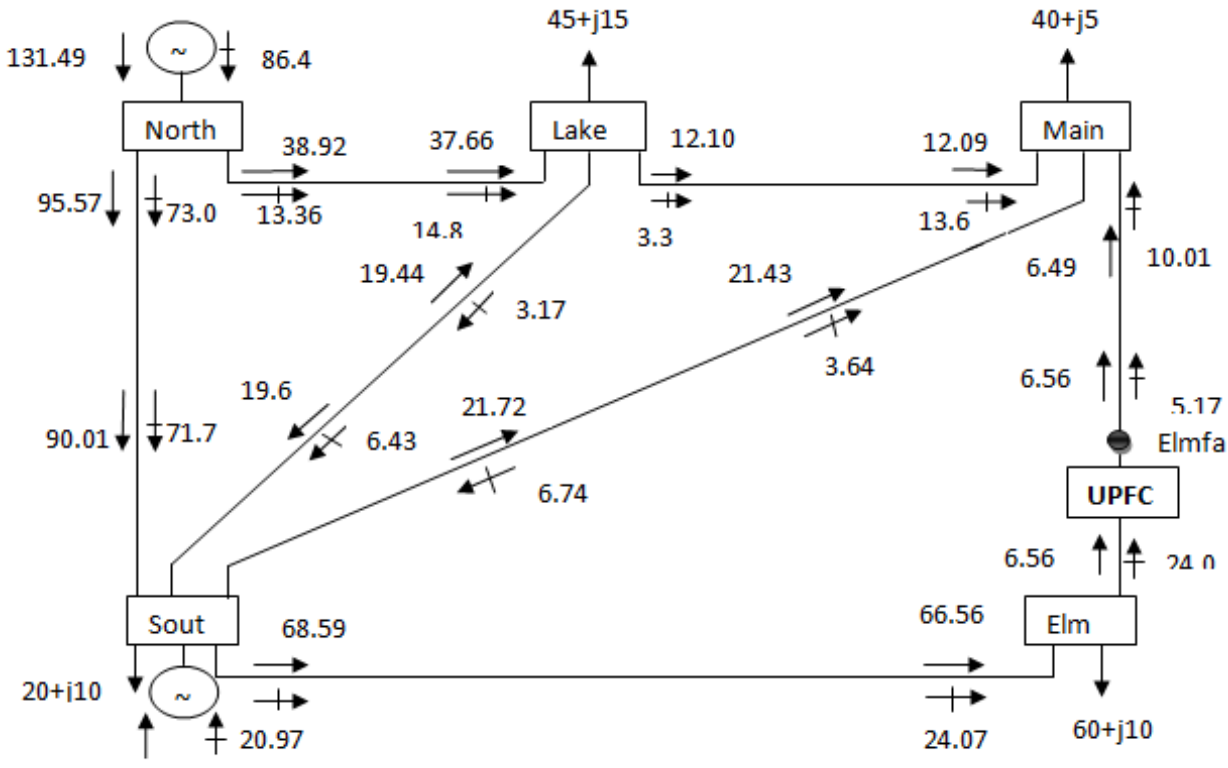

$$
\text { an } 75.49
$$

Fig. 5 The five-bus test network with one UPFC at Elm Bus, and the power flow results

Table 3 Voltage Profile for IEEE 5-bus network Connected with UPFC at Different Buses

\begin{tabular}{|l|l|l|l|l|}
\hline \multirow{3}{*}{ Bus No } & \multicolumn{2}{|l|}{ Exiting Method at Lake Bus } & \multicolumn{2}{l|}{ Proposed Method at Elm Bus } \\
\cline { 2 - 5 } & $\begin{array}{l}\text { Voltage } \\
\text { Mag(p.u) }\end{array}$ & $\begin{array}{l}\text { Voltage } \\
\text { Angle(deg) }\end{array}$ & $\begin{array}{l}\text { Voltage } \\
\text { Mag(p.u) }\end{array}$ & $\begin{array}{l}\text { Voltage } \\
\text { Angle(deg) }\end{array}$ \\
\hline North & 1.060 & 0.000 & 1.060 & 0.000 \\
South & 1.000 & -1.770 & 1.000 & -2.177 \\
Lake & 1.000 & -6.021 & 0.997 & -4.367 \\
Main & 0.992 & -4.997 & 0.996 & -4.590 \\
Elm & 0.972 & -5.77 & 1.000 & -7.346 \\
Elmfa & 0.997 & -2.51 & 1.020 & -4.053 \\
\hline \multicolumn{3}{|l|}{ Total Power Loss: } & Total Power Loss: \\
\multicolumn{2}{|l|}{ 12.329 MVA } & \multicolumn{3}{|l}{} \\
\hline
\end{tabular}


Application of Ga for Optimal Location and Parameters Setting Of Upfc Considering Voltage..

By considering the above Table 3, it is observed that voltage profile is improved and power losses in the network are reduced. Hence from the above Table 5.3 we concluded that from the voltage stability indices, the best location for UPFC can be determined.

The Genetic Algorithm control parameters selected are maximum generation (100), population size (10), Cross over probability (0.8) and Mutation Probability (0.004). The summary of results comparing conventional Newton-Raphson method with UPFC, GA is tabulated in Table 4.

Table 4 Summary of test results of IEEE 5-bus test system, UPFC located at Elm Bus.

\begin{tabular}{|l|l|l|}
\hline \multirow{2}{*}{ Aspect } & $\begin{array}{l}\text { Conventional Newton-Raphson } \\
\text { Method with UPFC }\end{array}$ & Proposed Method \\
\cline { 2 - 3 } $\begin{array}{l}\text { Total power loss without UPFC } \\
\text { (MVA) }\end{array}$ & 12.395 & Genetic Algorithm \\
\hline $\begin{array}{l}\text { Total power loss with UPFC } \\
\text { (MVA) }\end{array}$ & 12.100 & 12.395 \\
\hline $\begin{array}{l}\text { Cost of UPFC } \\
\text { (Rs/ kVAr) }\end{array}$ & 8118.994 & 12.097 \\
\hline $\begin{array}{l}\text { Net Saving } \\
\text { (Rs/Annum) }\end{array}$ & & 8208.227 \\
\hline & $86,99,196.021$ & $95,18,325.320$ \\
\hline Elapsed Time & $9.110320 \mathrm{Sec}$ & $7.478165 \mathrm{Sec}$ \\
\hline
\end{tabular}

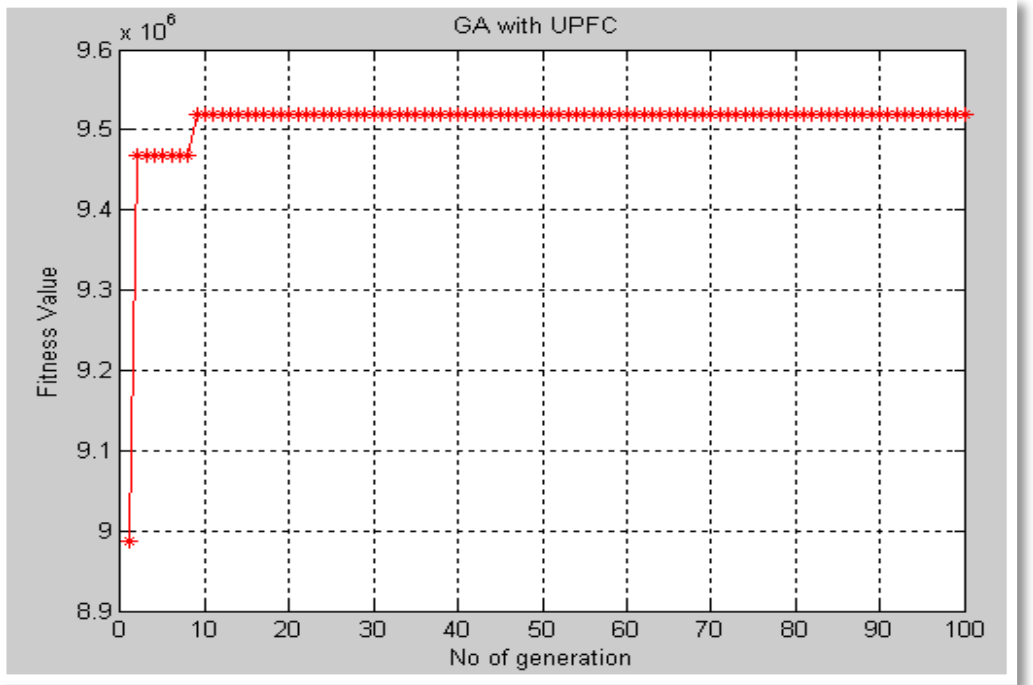

Fig. 6 Fitness function maximization by using GA for IEEE-5 bus test system, UPFC located at Elm Bus.

\subsection{Example-2: IEEE 14-Bus test system}

The problem of placement of the UPFC has also been solved of the IEEE 14-Bus test system. By considering the Voltage stability index $\left(L_{j}\right)$ value, it is observed that 14- Bus is more sensitive towards system security. Therefore 14-bus is more suitable location for UPFC to improve power system security/stability. Simulation results for Voltage magnitudes and phase angles without UPFC and with UPFC are shown in Table 5 , respectively.

Table 5 Conventional Newton-Raphson method without and with UPFC Voltage magnitudes, Phase Angles for IEEE 14-Bus test system

\begin{tabular}{|l|l|l|l|l|l|}
\hline \multicolumn{5}{|l|}{ Conventional Newton-Raphson Method } \\
\hline Without UPFC & With UPFC \\
\hline $\begin{array}{l}\text { Bus } \\
\text { Number }\end{array}$ & $\begin{array}{l}\text { Voltage Mag } \\
\text { (p. u) }\end{array}$ & Phase Angle (deg) & $\begin{array}{l}\text { Bus } \\
\text { Number }\end{array}$ & $\begin{array}{l}\text { Voltage Mag } \\
\text { (p. u) }\end{array}$ & Phase Angle (deg) \\
\hline 1 & 1.0600 & 0.000 & 1 & 1.0600 & 0.000 \\
\hline 2 & 1.000 & -4.411 & 2 & 1.000 & -4.413 \\
\hline 3 & 1.000 & -13.250 & 3 & 1.000 & -13.247 \\
\hline 4 & 0.982 & -10.270 & 4 & 0.985 & -10.330 \\
\hline 5 & 0.990 & -8.759 & 5 & 0.992 & -8.775 \\
\hline 6 & 1.000 & -15.344 & 6 & 1.000 & -15.143 \\
\hline 7 & 0.976 & -13.738 & 7 & 0.984 & -13.873 \\
\hline 8 & 1.000 & -13.738 & 8 & 1.000 & -13.873 \\
\hline
\end{tabular}


Application of Ga for Optimal Location and Parameters Setting Of Upfc Considering Voltage..

\begin{tabular}{|l|l|l|l|l|l|}
\hline 9 & 0.959 & -15.606 & 9 & 0.976 & -15.753 \\
\hline 10 & 0.958 & -15.886 & 10 & 0.972 & -15.972 \\
\hline 11 & 0.975 & -15.748 & 11 & 0.982 & -15.702 \\
\hline 12 & 0.982 & -16.309 & 12 & 0.982 & -16.076 \\
\hline 13 & 0.975 & -16.338 & 13 & 0.976 & -16.079 \\
\hline 14 & 0.946 & -17.117 & 14 & 0.959 & -16.647 \\
\hline
\end{tabular}

Table 6 Conventional Newton-Raphson method without and with UPFC Power flows for IEEE 14-Bus test system

\begin{tabular}{|c|c|c|c|c|c|c|}
\hline \multirow{2}{*}{$\begin{array}{l}\text { Line } \\
\text { number }\end{array}$} & \multirow{2}{*}{$\begin{array}{l}\text { From } \\
\text { Bus }\end{array}$} & \multirow[t]{2}{*}{ To Bus } & \multicolumn{2}{|c|}{$\begin{array}{l}\text { Conventional Newton Raphson without } \\
\text { UPFC }\end{array}$} & \multicolumn{2}{|c|}{$\begin{array}{lll}\text { Conventional Newton Raphson with } \\
\text { UPFC }\end{array}$} \\
\hline & & & $\begin{array}{l}\text { Active Power Loss } \\
\text { (p. u) }\end{array}$ & $\begin{array}{ll}\text { Reactive } & \text { Power } \\
\text { Loss (p. u) } & \\
\end{array}$ & $\begin{array}{l}\text { Active Power } \\
\text { Loss (p. u) }\end{array}$ & $\begin{array}{ll}\text { Reactive } & \text { Power } \\
\text { Loss (p. u) } & \\
\end{array}$ \\
\hline 1 & 1 & 2 & 0.0494 & 0.1227 & 0.0494 & 0.1228 \\
\hline 2 & 2 & 3 & 0.0270 & 0.0917 & 0.0269 & 0.0915 \\
\hline 3 & 2 & 4 & 0.0178 & 0.0358 & 0.0181 & 0.0365 \\
\hline 4 & 1 & 5 & 0.0301 & 0.0984 & 0.0300 & 0.0979 \\
\hline 5 & 2 & 5 & 0.0099 & 0.0133 & 0.0099 & 0.0133 \\
\hline 6 & 3 & 4 & 0.0059 & 0.0019 & 0.0055 & 0.0029 \\
\hline 7 & 4 & 5 & 0.0051 & 0.0098 & 0.0053 & 0.0104 \\
\hline 8 & 5 & 6 & 0 & 0.0522 & 0 & 0.0488 \\
\hline 9 & 4 & 7 & 0.0001 & 0.0170 & 0.0001 & 0.0177 \\
\hline 10 & 7 & 8 & 0 & 0.0033 & 0 & 0.0014 \\
\hline 11 & 4 & 9 & 0 & 0.0157 & 0 & 0.0156 \\
\hline 12 & 7 & 9 & 0 & 0.0117 & 0 & 0.0100 \\
\hline 13 & 9 & 10 & 0.0001 & 0.0002 & 0.0001 & 0.0003 \\
\hline 14 & 6 & 11 & 0.0013 & 0.0028 & 0.0008 & 0.0017 \\
\hline 15 & 6 & 12 & 0.0009 & 0.0019 & 0.0009 & 0.0018 \\
\hline 16 & 6 & 13 & 0.0028 & 0.0056 & 0.0026 & 0.0052 \\
\hline 17 & 9 & $14 / 15$ & 0.0011 & 0.0024 & 0.0037 & 0.0079 \\
\hline 18 & 10 & 11 & 0.0006 & 0.0013 & 0.0002 & 0.0005 \\
\hline 19 & 12 & 13 & 0.0001 & 0.0001 & 0.0001 & 0.0001 \\
\hline 20 & 13 & 14 & 0.0011 & 0.0023 & 0.0009 & 0.0019 \\
\hline
\end{tabular}

The IEEE 14-Bus test network is modified to include one UPFC to compensate the transmission line link Bus-14 and Bus-15. The UPFC is used to maintain active and reactive powers leaving the UPFC, towards 9-Bus at 9.9 MW and 0.19 MVAR, respectively. Moreover the UPFC shunt converter is set to regulate the nodal voltage magnitude at Bus 15 at $1 \mathrm{p}$. u. The starting values of the UPFC voltage sources are taken to be $V_{c R}=$ 0.0099 p.u, $\delta_{c R}=-89.978^{0}, V_{v R}=1 p . u$ and $\delta_{v R}=0^{0}$. The source impedances have values of $X_{c R}=X_{v R}=$ 0.1 p.u. Convergence is obtained in tenth iteration to a power mismatch tolerance $1 e^{-12}$. The UPFC held its target values. The power flow results are shown in above Table 6.

The Genetic Algorithm control parameters selected are maximum generation (100), population size (10), Cross over probability (0.8) and Mutation Probability (0.004). Simulation results for voltage magnitudes, phase angles; active and reactive power losses with UPFC in the system for Genetic Algorithm (GA) are shown in Table 7 , respectively.

Table 7 Voltage magnitudes, Phase Angles; Active and Reactive power losses with UPFC by using GA

\begin{tabular}{|l|l|l|l|l|l|l|l|}
\hline $\begin{array}{l}\text { Line } \\
\text { Number }\end{array}$ & $\begin{array}{l}\text { From } \\
\text { Bus }\end{array}$ & To Bus & $\begin{array}{l}\text { Active Power Loss } \\
\text { (p. u) }\end{array}$ & $\begin{array}{l}\text { Reactive Power } \\
\text { Loss (p. u) }\end{array}$ & $\begin{array}{l}\text { Bus } \\
\text { Number }\end{array}$ & $\begin{array}{l}\text { Voltage } \\
\text { Mag (p. u) }\end{array}$ & $\begin{array}{l}\text { Phase } \\
\text { Angle (deg) }\end{array}$ \\
\hline 1 & 1 & 2 & 0.0494 & 0.1228 & 1 & 1.060 & 0.000 \\
\hline 2 & 2 & 3 & 0.0269 & 0.0915 & 2 & 1.000 & -4.414 \\
\hline 3 & 2 & 4 & 0.0181 & 0.0364 & 3 & 1.000 & -13.249 \\
\hline 4 & 1 & 5 & 0.0300 & 0.0980 & 4 & 0.985 & -10.329 \\
\hline 5 & 2 & 5 & 0.0099 & 0.0134 & 5 & 0.992 & -8.783 \\
\hline 6 & 3 & 4 & 0.0055 & 0.0029 & 6 & 1.000 & -15.224 \\
\hline 7 & 4 & 5 & 0.0052 & 0.0102 & 7 & 0.984 & -13.835 \\
\hline 8 & 5 & 6 & 0.0000 & 0.0499 & 8 & 1.000 & -13.835 \\
\hline 9 & 4 & 7 & 0 & 0.0173 & 9 & 0.976 & -15.696 \\
\hline 10 & 7 & 8 & 0.0000 & 0.0014 & 10 & 0.972 & -15.940 \\
\hline 11 & 4 & 9 & 0.0000 & 0.0153 & 11 & 0.982 & -15.725 \\
\hline 12 & 7 & 9 & 0.0000 & 0.0099 & 12 & 0.982 & -16.187 \\
\hline 13 & 9 & 10 & 0.0001 & 0.0003 & 13 & 0.975 & -16.216 \\
\hline 14 & 6 & 11 & 0.0008 & 0.0016 & 14 & 0.948 & -16.983 \\
\hline 15 & 6 & 12 & 0.0009 & 0.0019 & 15 & 1.000 & -18.141 \\
\hline 16 & 6 & 13 & 0.0028 & 0.0055 & & &
\end{tabular}


Application of Ga for Optimal Location and Parameters Setting Of Upfc Considering Voltage..

\begin{tabular}{|l|l|l|l|l|}
\hline 18 & 10 & 11 & 0.0002 & 0.0005 \\
\hline 19 & 12 & 13 & 0.0001 & 0.0001 \\
\hline 20 & 13 & 14 & 0.0011 & 0.0022 \\
\hline
\end{tabular}

Simulation results for voltage magnitudes, phase angles, active and reactive power losses with UPFC in the system for Genetic Algorithm (GA) are shown in Table 7, respectively. The summary of results comparing with the existing method, GA is tabulated in Table 8.

Table 8 Summary of results of IEEE-14 Bus test system

\begin{tabular}{|l|l|l|}
\hline \multirow{2}{*}{ Aspect } & $\begin{array}{l}\text { Conventional Newton-Raphson } \\
\text { Method with UPFC }\end{array}$ & Proposed Method \\
\cline { 2 - 3 } & Genetic Algorithm \\
\hline Total power loss without UPFC (MVA) & 50.992 & 50.992 \\
\hline Total power loss with UPFC (MVA) & 8634.701 & 50.633 \\
\hline Cost of UPFC (Rs/kVAr) & & 8643.865 \\
\hline Net Saving(Rs/Annum) & $1,02,17,769.757$ & $1,08,75,276.908$ \\
\hline Elapsed Time & $21.100820 \mathrm{Sec}$ & $17.850077 \mathrm{Sec}$ \\
\hline
\end{tabular}

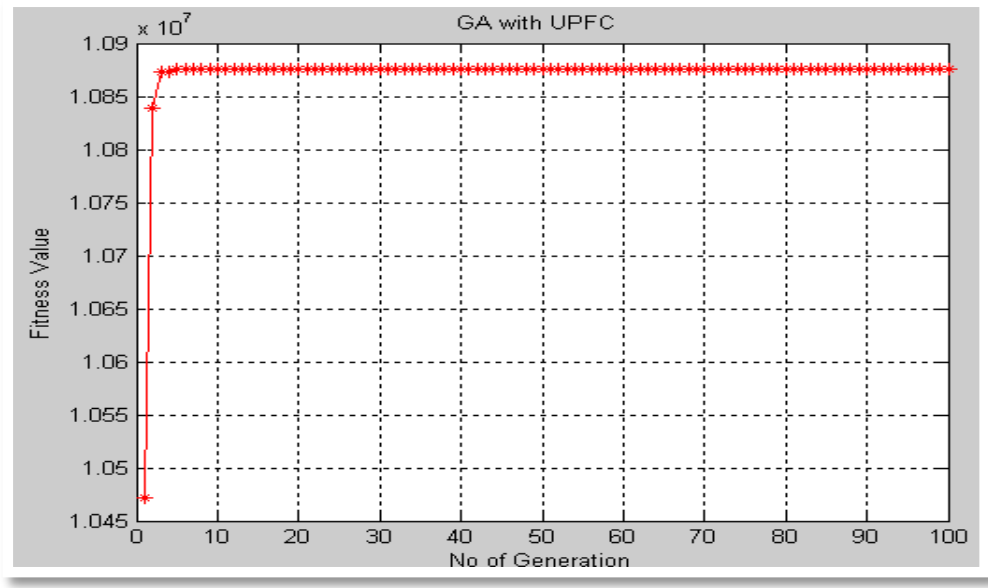

Fig. 7 Fitness function maximization by using GA for IEEE-14 Bus test system.

The proposed algorithms were implemented to find out the proper setting and installation cost of the UPFC in IEEE-5 Bus and IEEE-14 Bus test system. Comparisons of two proposed algorithms are shown in Tables 4, 8 and 5, From Figs. 6-7, it is observed that fitness function is maximization in GA. Tables 4, 7 shows that GA is faster than Conventional Method from the perspective of time and this is due to the purpose that GA has selection, crossover and mutation operations. The simulation studies were carried out on Pentium IV, 1.60 GHz, 1GB RAM in MATLAB 7.1 environment.

\section{Conclusion}

In this paper, the significant results obtained during the course of the work are presented and a few suggestions for future research are presented. The power flow analysis with the inclusion of UPFC has been analyzed. Newton-Raphson method used in polar co-ordinate form is effectively applied to solve the power flow equation of IEEE 5-Bus and IEEE 14-Bus systems which differ from each other in size and degree of operational complexity. The UPFC model is incorporated into an existing Newton-Raphson load flow algorithm, which is capable of solving large power networks very reliably. It shows that UPFC can be set to control active and reactive powers and voltage magnitude simultaneously. The voltage stability index is used to find location of the UPFC in the network.

The proposed algorithms were implemented to find out the proper setting and installation cost of the UPFC in IEEE-5 Bus and IEEE-14 Bus test system. By comparing the results, it is observed that GA is more effective than Conventional Method in terms of fitness function. Moreover, the time required for execution is less for GA than Conventional Method.

\section{References}

[1]. Chandrabhan Sharma and Marcus G. Ganness, "Determination of power system voltage stability using modal analysis", POWERENG 2007, pp.381-386, April 2007.

[2]. G. Wu, A.Yokoyama, J. He and Y.Yu,"Allocation and control of FACTS devices for steady-state stability enhancement for largescale power system,"IEEE International Conference on Power System Technology, Vol.1, pp. 357-361, August 1998. 
[3]. H. Okamoto, A. Kurita, Y.Sekine,"A method for identification of effective locations of variable impedence apparatus on enhancement of steady-state stability in large scale power systems", IEEE Transactions on Power Systems,Vol.10,No.3,pp.14011407,August 1995

[4]. N. K. Sharma, A.Ghosh and R. K. Varma, "A novel placement strategy for FACTS controllers", IEEE Transactions on Power Delivery, Vol.18, no.3, pp.982-987, July 2003.

[5]. Nuraddeen Magaji, and M. W. Mustafa," Optimal location of FACTS devices for damping oscillations using Residue factor", IEEE International Conference on Power and Energy, pp.1339- 1344, December 2008.

[6]. N. G. Hingorani and L. Gyugyi, "Understanding FACTS-concepts and technology of flexible AC transmission systems," IEEE press, First Indian Edition, 2001.

[7]. K. P. Wang, J. Yurevich, A. Li, "Evolutionary- programming-based load flow algorithm for systems containing unified power flow controllers," IEE Proc.-Gener. Transm .Distribute Vol.150, No. 4, Jul. 2003.

[8]. Paserba, N.Miller, E.Laesen and R.Piwko," A Thyristor controlled series compensation model for power system stability analysis," IEEE Trans.on Power-Delivery, vol. 10, pp.1471-1478, 1995.

[9]. N. G. Hingorani, "Power electronics in electrical utilities: role of power electronics in future power systems," Proceedings of the IEEE Vol. 76 No.4, pp.481-482, Apr. 1988

[10]. T.T. Lie and W. Deng, "Optimal Flexible AC transmission systems (FACTS) devices allocation”, Electr. Power Energy Systems. 19 (2), 1999, pp. 125-134.

[11]. G. W. Stagg, and A. H. El-Abiad, Computer Methods in Power System Analysis, McGraw-Hill, 1968. 